\title{
Cell-Based Broadcasting Algorithms in Mobile Ad-Hoc Networks
}

\author{
Abderezak Touzene, Khaled Day, Bassel Arafeh, Nasser Alzeidi \\ Computer Science department, Sultan Qaboos University, Oman \\ touzene@squ.edu.om, kday@squ.edu.om, arafeh@squ.edu.om, \\ alzidiesqu.edu.om
}

\begin{abstract}
This paper proposes new cell-based broadcasting algorithms (CBB) for mobile ad-hoc networks (MANETS). It shows how communication methods originally designed for wired multiprocessor interconnection networks can be used in MANETs. CBB algorithms are based on a logical 2-dimensional grid view of the geographical region of the MANET. They make use of existing spanning trees in the 2dimensional grid interconnection networks to support broadcasting in MANETs. In this study we developed a simulation model to measure the delivery ratio and the number of rebroadcast messages and compare the results with the well known probabilistic broadcasting algorithm.
\end{abstract}

\section{KEYWORDS}

Interconnection Networks; Mobile ad-hoc Networks; Broadcasting; Position-based routing; Spanning trees.

\section{INTRODUCTION}

A Mobile Ad Hoc Network (MANET) is a collection of wireless nodes communicating with each other in the absence of any fixed infrastructure. MANET is a developing area of research. Efforts have been taken for achieving efficient broadcasting in mobile ad hoc networks. Network broadcasting is the process in which one node sends a packet to all other nodes in the network. Numerous network protocols are based on broadcasting: routing, information dissemination, and service/resource discovery. Since many systems have stringent end-to-end delay requirements, the design of low-latency and low overhead broadcasting schemes is essential to many practical applications.

A broad category of routing and broadcast protocols is the class of position-based protocols [1-4]. These protocols make use of the nodes' geographical positions to make routing decisions. Nodes are able to obtain their own geographical position via Global Positioning System (GPS). This approach has become practical by the rapid development of hardware and software solutions for determining absolute or relative node positions in MANETs [5].

In this paper we propose a new cell-based broadcast protocol for MANETs called the CBB protocol. In CBB protocol each node knows only its position using the GPS system. CBB protocol uses a logical grid view of the geographical region and makes use of known spanning trees of the grid interconnection network [6-7] to perform broadcasting over the logical view. The

DOI : 10.5121/jgraphoc.2011.3401 
International journal on applications of graph theory in wireless ad hoc networks and sensor networks

(GRAPH-HOC) Vol.3, No.4, December 2011

geographical region of the MANET is viewed as a 2-dimensional grid of cells as shown in Fig. 1. With the help of a spanning tree rooted at a given cell in the grid, a node called the initiator of the broadcast message within the root cell, broadcasts its message to neighbouring nodes in neighbouring cells (neighbour cell in the spanning tree of cells). As a matter of fact this first step is similar to other broadcasting algorithms, such as those for flooding or probabilistic broadcast. In the second step of the CBB protocol, only one node (gateway node) per cell rebroadcasts the message. In flooding algorithm, all the nodes which receive the message will rebroadcast it (broadcast storm problem) or few nodes will rebroadcast the message based on a specified probability to reduce the effect of broadcast storm in the probability based broadcast algorithms. In CBB, the problem of broadcasting in mobile MANET nodes is transformed into a problem of broadcasting in geographically fixed grid cells using only the gateway nodes as forwarders. A packet is forwarded from a node in a grid cell to nodes in neighbouring grid cells (in the spanning tree) repeatedly until it reaches all nodes in the leaf cells of the spanning tree.

The remainder of the paper is organized as follows: section 2 presents the related works; section 3 presents assumptions and notations; section 4 presents an overview of the proposed CBB protocol; section 5 gives a detailed implementation of $\mathrm{CBB}$ protocol; section 6 presents the reliability and performance of $\mathrm{CBB}$ and section 7 concludes the paper.

\section{Related Works}

The idea of treating the geographic area as a number of logical grids, each as a number of logical square grid has been used first in [15] to develop link-state routing, and then in [13], to develop location-aware routing. In [13], one mobile host in each grid (if any) is elected as the leader of the grid. The routing algorithm called GRID is performed in a cell-by-cell manner through the grid leaders. GRID is a routing algorithm it is not a broadcasting algorithms. In [17], they propose an efficient service discovery protocol based on grid architecture for MANETs called SGrid. The geographical area of a MANET is also treated as a 2D logical grid. This work proposes a hierarchical mechanism for identifying its grid level. One node in its grid cell is elected as a directory for caching available service descriptions. In CBB protocol is a broadcasting algorithm, which use a similar idea as in [13][17], but the difference is that within a cell there might be more than one cell leader (for different forward directions) and more importantly we do not have any election to select the leader nodes, which reduce substantially the cost of leader selection. The leaders (we call them gateways) selection procedure is described in the next section. To the best of our knowledge CBB is the first proposed algorithm based on grid, which use spanning trees, where the nodes represent the grid cells of the $2 \mathrm{D}$ grid region.

In [8], broadcast algorithms in MANET were categorized into four families: Simple Flooding, Probability Based Methods, Area Based Methods and Neighbour Knowledge Methods. We briefly give an overview of each category.

\subsection{Simple Flooding}

A Simple Flooding $[9,10]$ starts with a source node broadcasting a packet to all neighbours. Each of those neighbours in turn rebroadcasts the packet exactly one time and this continues until all reachable network nodes have received the packet. The advantages of this technique is its high reliability (high delivery ratio) especially when the MANET has low density and high mobility. The disadvantages are high number of rebroadcasts, which increase the probability of collisions at the MAC layer and also increases the broadcast time for moderate and high density MANETs. 
International journal on applications of graph theory in wireless ad hoc networks and sensor networks

(GRAPH-HOC) Vol.3, No.4, December 2011

\subsection{Probability Based Methods}

Probabilistic Scheme: The Probabilistic scheme from [11] is similar to Flooding, except that nodes only rebroadcast with a predetermined probability $p$. Randomly having some nodes not rebroadcast saves node and network resources without harming delivery effectiveness. When the probability is 1 , this scheme is identical to Flooding. Because not all nodes rebroadcast would improve the broadcast time (less collision). In other hands, when $p$ is small and the MANET density is low then the delivery ratio might be affected.

We will compare the performance of our CBB protocol with the performance of this probabilistic scheme.

Counter-Based Scheme: $\mathrm{Ni}$ et al [11] show an inverse relationship between the number of times a packet is received at a node and the probability of that node being able to reach additional area on a rebroadcast. This result is the basis of their Counter-Based scheme. Upon reception of a previously unseen packet, the node initiates a counter with a value of one and sets a random number, "Random Assessment Delay" RAD (which is randomly chosen between 0 and Tmax seconds). During the RAD, the counter is incremented by one for each redundant packet received. If the counter is less than a threshold value when the RAD expires, the packet is rebroadcast. Otherwise, it is simply dropped. From [11], threshold values above six relate to little additional coverage area being reached. The advantage of this technique is its adaptability to local topologies. That is, in a dense area of the network, some nodes would not rebroadcast; in sparse areas of the network, more nodes rebroadcast.

\subsection{Area Based Methods}

Suppose a node receives a packet from a sender that is located only one meter away. If the receiving node rebroadcasts, the additional area covered by the retransmission is quite low. On the other extreme, if a node is located at the boundary of the sender node's transmission distance, then a rebroadcast would reach significant additional area [11]. A node using an Area Based Method can evaluate additional coverage area based on all received redundant transmissions during a random interval, RAD period. This technique supposes that each node knows its own position using a GPS and the initial node or rebroadcast node adds its location to the header of the broadcast message which will make distance calculation easy between sender and receiver nodes.

\subsection{Knowledge Based Methods}

The Neighbour Knowledge Methods is what Lim and Kim refer to as Flooding with Self Pruning [12]. This protocol requires that each node have knowledge of its 1-hop neighbours. A node includes its list of known neighbours in the header of each broadcast packet. A node receiving a broadcast packet compares its neighbour list to the sender's neighbour list. If the receiving node would not reach any additional nodes, it refrains from rebroadcasting; otherwise the node rebroadcasts the packet. Other variations require knowledge of its 1-hop and 2-hops neighbours. Clearly all the presented classes of broadcast algorithms in the MANETs try to reduce as much as possible the number of rebroadcast, which will have a positive impact on the performance of the algorithm. 
International journal on applications of graph theory in wireless ad hoc networks and sensor networks

(GRAPH-HOC) Vol.3, No.4, December 2011

\section{Preliminaries}

We assume a mobile ad hoc network (MANET) is composed of $N$ mobile wireless devices (nodes) distributed in a given geographical region. We view the geographical region where the nodes are located as a logical $k \times k$ two-dimensional (2D) grid of cells as shown in Fig.1. The length of a side of a grid cell is denoted by $d$. Two grid cells are called neighbours if they have a common side. Therefore each grid cell has 8 neighbouring cells except for cells located at the boundaries of the grid which have 2 or 3 neighbouring cells each. A path in the 2D-grid is a sequence of neighbouring grid cells.

Two MANET nodes are called neighbouring nodes if they are located in neighbouring cells. The value of $d$ is selected depending on the transmission range $r$ such that a MANET node located at any position within a grid cell can communicate directly with all its neighbouring nodes (located in 8 neighbouring grid cells). This requirement is met if $d$ satisfies: $r \geq 2 d \sqrt{2}$. This can be seen by noticing that the farthest apart points in two adjacent grid cells are two diametrically opposite corners at distances $2 d$. (See figure 2, [13])

We set $d$ to the limit $r / 2 \sqrt{2}$ in order to minimize the number of broadcast steps. Each grid cell is identified by a pair of integer grid coordinates $(x, y)$ as illustrated in Figure 1. Each MANET node has a distinctive node id (IP or MAC address). We assume nodes are able to obtain their own geographical positions through a low-power GPS receiver.

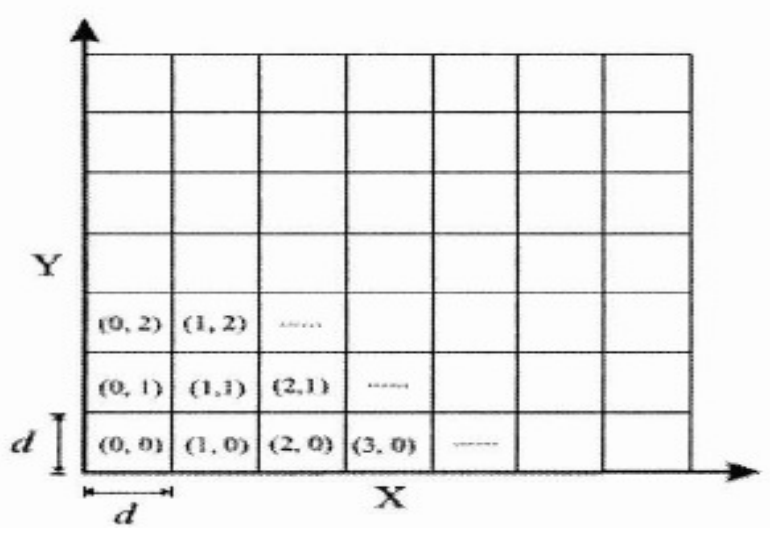

Figure 1: Logical 2D-Grid View of the MANET Area

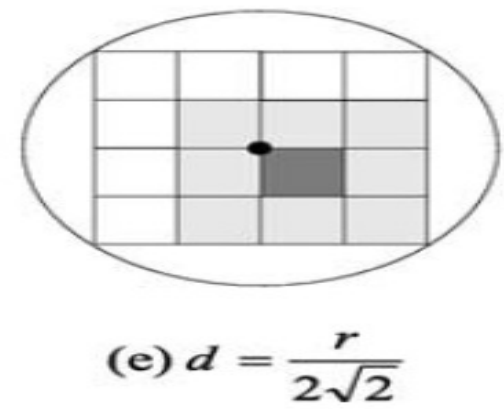

Figure 2: Cell Neighborhood in the 2D-Grid 
International journal on applications of graph theory in wireless ad hoc networks and sensor networks

(GRAPH-HOC) Vol.3, No.4, December 2011

\section{OVERVIEW OF THE CBB BROADCAST PROTOCOL}

The advantage of using a spanning tree of the grid cells ensures each grid cell will receive only one copy of the broadcast message by the gateway node of this cell and it is also heard by all the nodes within the same cell (we assume promiscuous mode is used). Only the gateway node of the cell will rebroadcast the message to the next cell in the spanning tree. In CBB protocol, when the broadcast message is received by nodes located in a given grid cell only one node will rebroadcast the message. Having only one node acting as a rebroadcast node in the CBB solves the broadcast storm problem and reduces the number of messages in the MANET. CBB protocol has three major phases: Initialization Phase, Forward Phase and Maintenance phase.

\subsection{Initialization Phase}

In CBB protocol each node maintains a list of gateway nodes (one in each of the four directions: $x+, x-, y+, y$-). As an example, for a node located at the cell $(x, y), x+$ will store the ID of the node selected as the gateway node in the neighbour cell $(x+1, y)$. $x$-, will store the ID of the node selected as the gateway node in the neighbour cell $(x-1, y)$. Similarly the gateways $y+, y$ - which will store neighbour gateways respectively located in the cells $(x, y+1)$ and $(x, y-1)$.

Initially, to update the list of gateways for all the nodes, each node sends a "HELLO" message to its neighbourhood in order to discover its gateways on the four directions. When a node receives a "HELLO" message it replies as a potential gateway node in that direction. The initiator of the "HELLO" message selects the latest sender of reply in a specific direction as a gateway for that direction.

\subsection{Forwarding Phase}

At the reception of a broadcast message if the node is a gateway node, it will rebroadcast the message to the next cell along the spanning tree (see the next section) using its gateway information in the direction of that specific cell. If the node is not a gateway node, it consumes the message.

\subsection{Maintenance Phase}

A node continuously updates its list of gateway nodes as it communicates with nodes in neighbouring cells. Every time a node receives a message (unicast or broadcast) from a node in a certain direction it sets that node as its new gateway node in that direction. This avoids gateway election overheads as in [13] or cluster heads [14]. In CBB protocol we assume that unicast routing, and more than one broadcast operations can happen at the same time.

When a node exits a cell it sends a cell exit message. If a node exits a cell and enters a new cell, the current information about its gateways is not consistent. The node has to perform a new Initialization Phase in order to update its list of gateways. Upon the reception of exit from $(x, y)$ cell message each node within the cell $(x, y)$ has to perform a new Initialization Phase to tell the neighbourhood of the exited node to update their list of gateways (the exited node might be in the list of their gateways). 
International journal on applications of graph theory in wireless ad hoc networks and sensor networks

(GRAPH-HOC) Vol.3, No.4, December 2011

\section{DETAILS OF THE CBB BROADCAST PROTOCOL}

We present in this section a detailed implementation of the proposed broadcast protocol using a spanning tree of the grid as shown in Fig. 3 (the black circles correspond to the gateway nodes in the cells).

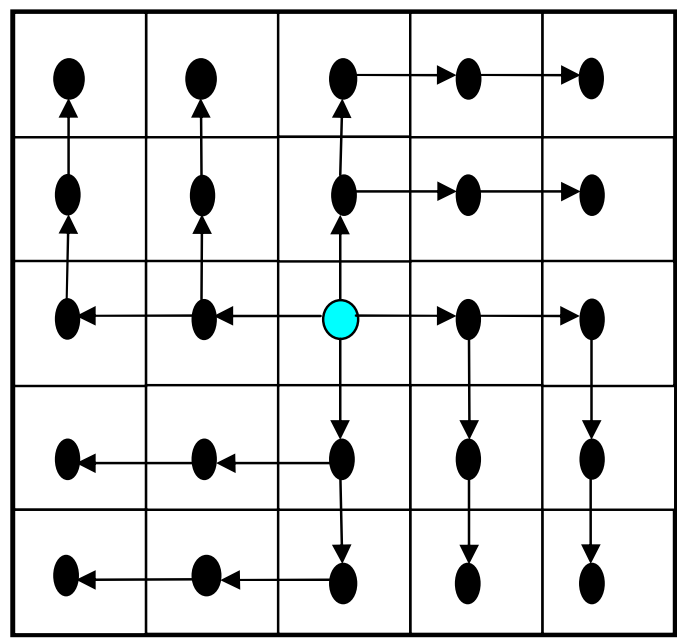

Figure 3.a : Spanning Tree of the 2D-Grid T1

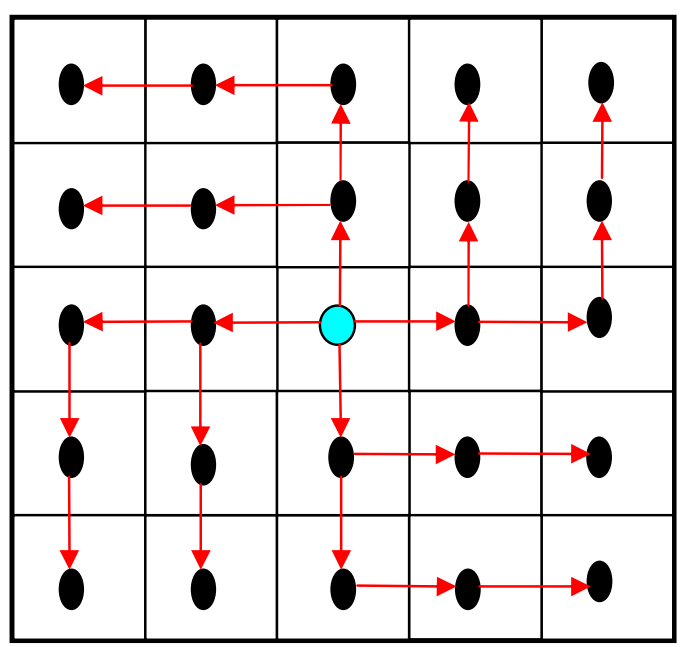

Figure 3.b: A Second Spanning Tree of the 2D-Grid T2 
International journal on applications of graph theory in wireless ad hoc networks and sensor networks (GRAPH-HOC) Vol.3, No.4, December 2011

The following broadcasting algorithm uses T1 spanning tree:

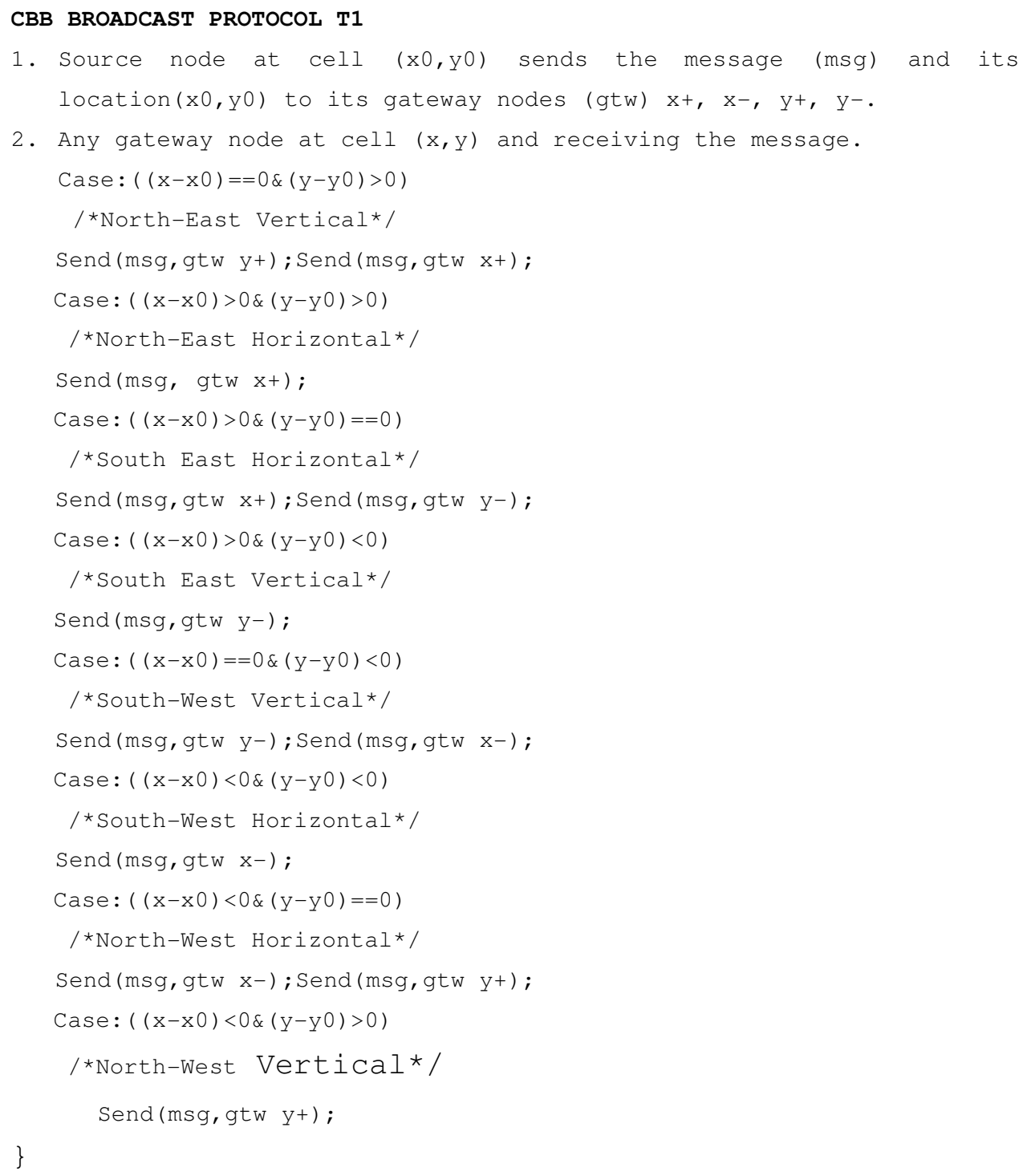


International journal on applications of graph theory in wireless ad hoc networks and sensor networks (GRAPH-HOC) Vol.3, No.4, December 2011

The following broadcasting algorithm uses $\mathrm{T} 2$ spanning tree:

\section{CBB BROADCAST PROTOCOL T2}

1. Source node at cell $(\mathrm{x} 0, \mathrm{y} 0)$ sends the message $(\mathrm{msg})$ and its location $(\mathrm{x} 0, \mathrm{y} 0)$ to its gateway nodes $(\mathrm{gtw}) \mathrm{x}+, \mathrm{x}-, \mathrm{y}+, \mathrm{y}-$.

2. Any gateway node at cell $(\mathrm{x}, \mathrm{y})$ and receiving the message.

Case: $((\mathrm{x}-\mathrm{x} 0)==0 \&(\mathrm{y}-\mathrm{y} 0)>0)$

/*North-West Vertical*/

Send(msg,gtw y+);Send(msg,gtw x-);

Case: $((\mathrm{x}-\mathrm{x} 0)>0 \&(\mathrm{y}-\mathrm{y} 0)>0)$

/*North-East Vertical*/

Send(msg, gtw y+);

Case: $((\mathrm{x}-\mathrm{x} 0)>0 \&(\mathrm{y}-\mathrm{y} 0)==0)$

/*North East Horizontal*/

Send(msg,gtw $\mathrm{x}+$ ); Send(msg,gtw y+);

Case: $((\mathrm{x}-\mathrm{x} 0)>0 \&(\mathrm{y}-\mathrm{y} 0)<0)$

/*South East Horizontal*/

Send(msg,gtw $\mathrm{x}+)$;

Case: $((x-x 0)==0 \&(y-y 0)<0)$

/*South-East Horizantal*/

Send(msg,gtw y-);Send(msg,gtw x+);

Case $:((\mathrm{x}-\mathrm{x} 0)<0 \&(\mathrm{y}-\mathrm{y} 0)<0)$

/*South-West Vertical*/

Send(msg,gtw y-);

Case: $((\mathrm{x}-\mathrm{x} 0)<0 \&(\mathrm{y}-\mathrm{y} 0)==0)$

/*South-West Horizontal*/

\section{Performance Evaluation of CBB Protocols}

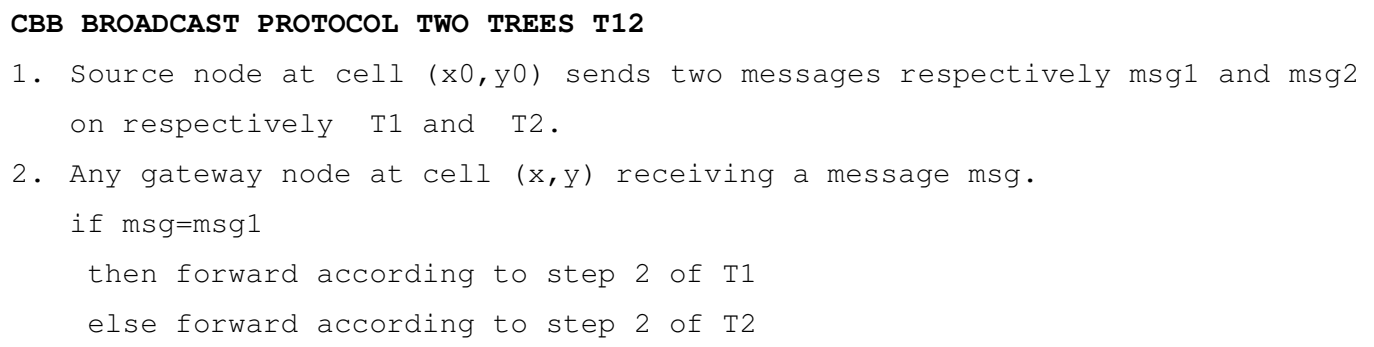


International journal on applications of graph theory in wireless ad hoc networks and sensor networks

(GRAPH-HOC) Vol.3, No.4, December 2011

\subsection{Analytical Performance of CBB}

To measure the performance of the CBB broadcast we estimate the number of rebroadcast messages $(N B R)$ in the MANET from the start of the broadcast operation until its end. We use $N B R$ to compare with other broadcast protocols such as the probabilistic broadcast protocol PROB. We consider the worst-case analysis: each cell has at least one node and the source node is located at one of the four corner cells. In this case all the gateway nodes (one per cell) except the ones located in opposite side grid cell in the last row and last column The total number of messages generated by $\mathrm{CBB}$ protocol is:

$$
N B R_{C B B}=(k-1)^{2} .
$$

For the probabilistic broadcast protocol PROB at the first step one message is broadcasted. Assume that 3 neighbouring cells (sender located in one of the corner cells) have received the message. If we assume that the network density is $D$ hosts per cell, and the rebroadcast probability is $P$, then 3.D.P new messages will be rebroadcasted in the next step then $(5 D P)$ will be rebroadcasted and so on. At the step $i,(2 i+1)$.D.P nodes will rebroadcast. Since the sum of the first $k$ odd integer number is $k^{2}$, the total amount of messages until the broadcast ends is:

$$
N B R_{P R O B}=k^{2} . D \cdot P
$$

Note that $N B R_{C B B}$ is independent from the network density $D$, which is not the case for $N B R_{P R O B}$. We can express $N B R_{P R O B}$ in terms of $D$ as follows:

$$
N B R_{P R O B}=N \cdot D^{2} \cdot P
$$

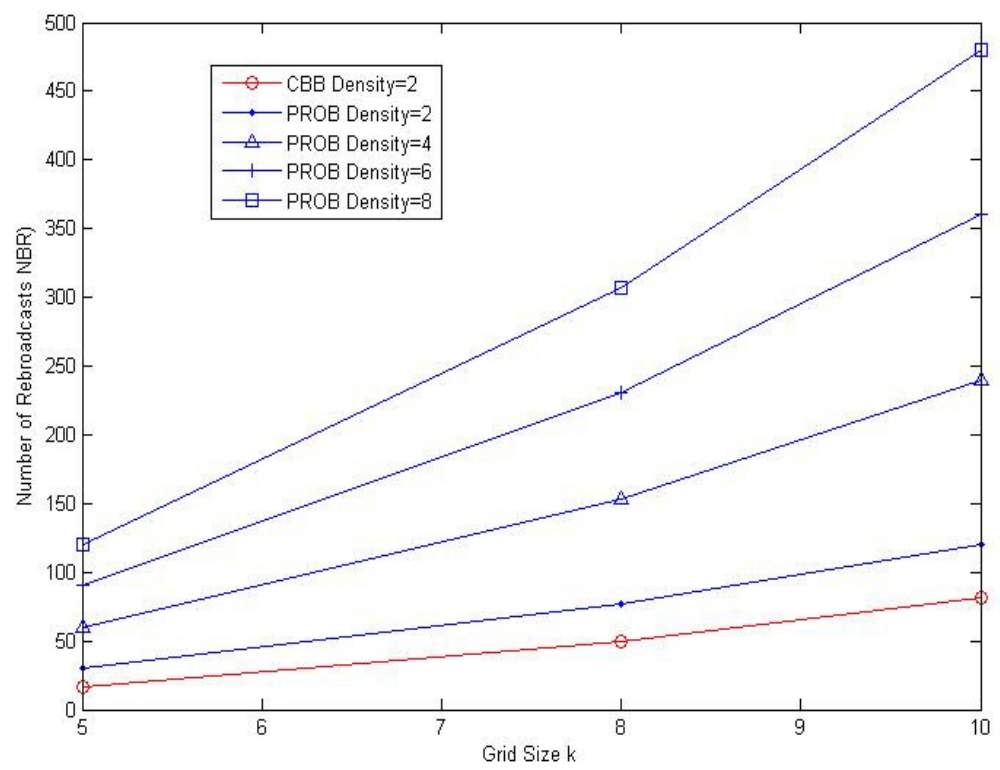

Figure 4: Analytic NBR varying the MANET Density and Size 
International journal on applications of graph theory in wireless ad hoc networks and sensor networks

(GRAPH-HOC) Vol.3, No.4, December 2011

Figure 4, shows that the number of rebroadcast messages in CBB is much lower (less collision at the data link layer) than in PROB, which increase quadratically in term of D.

\subsection{Performance of CBB Using Simulation Model}

We have developed a simulation model based on MATLAB 7.8 to evaluate and compare the performance of the CBB and PROB protocols. The MANET covers a physical area of size $500 \mathrm{~m}$ $\times 500 \mathrm{~m}$ with a number of nodes varying from 50 to 200 mobile hosts.

The simulation runs for $500 \mathrm{~s}$. The results were obtained from an average of 10000 broadcast operations. The mobility model used is the Random Waypoint [16]. A node chooses one random destination in the simulation area. Then, it moves to that destination at a random speed (varying from $5 \mathrm{Km} / \mathrm{H}, 20 \mathrm{Km} / \mathrm{H}$, and $50 \mathrm{Km} / \mathrm{H}$ ). Upon reaching its destination, it selects another random

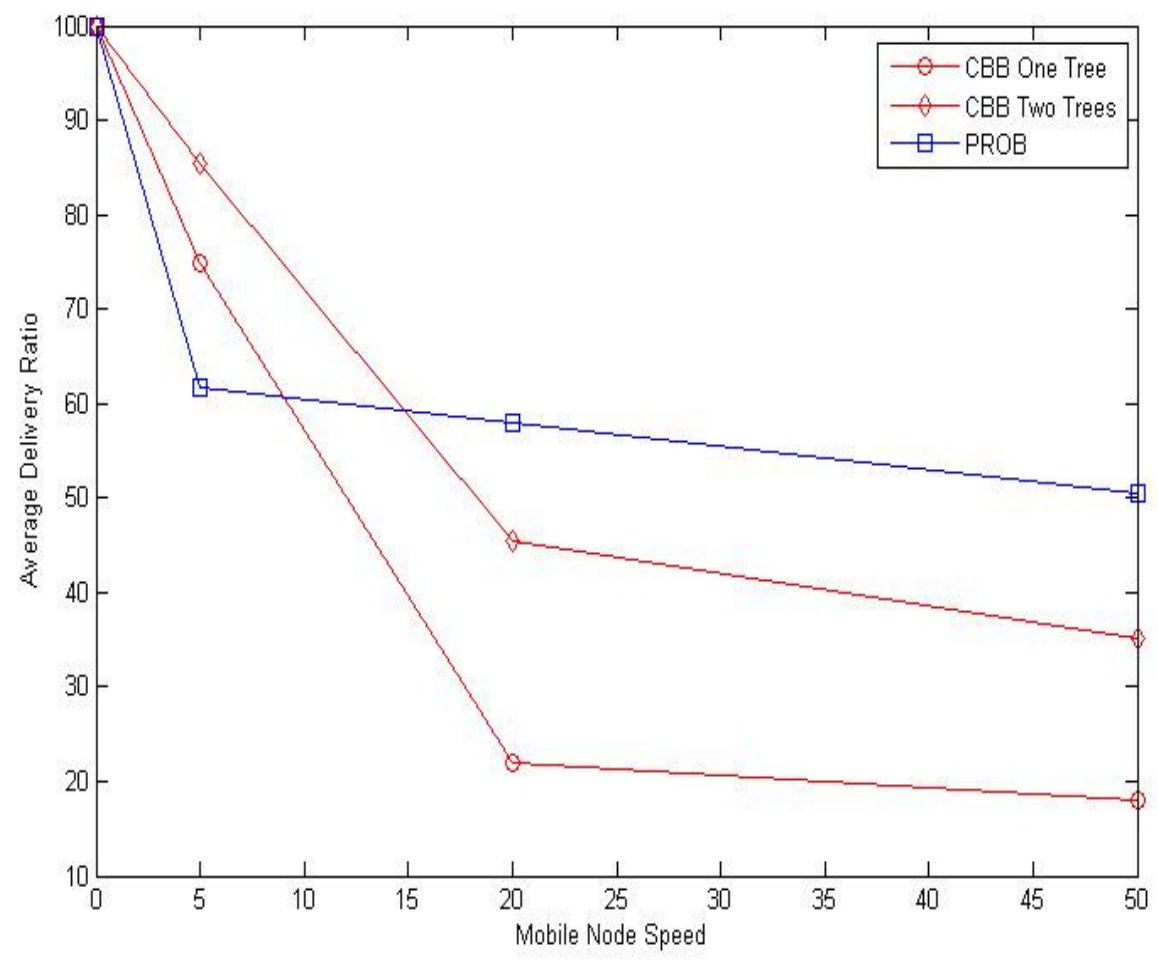

destination (pause time $=0$ ) inside the simulation area, and proceeds as previously described.

Figure 5: Delivery Ratio for Density=1 versus Mobility 
International journal on applications of graph theory in wireless ad hoc networks and sensor networks

(GRAPH-HOC) Vol.3, No.4, December 2011

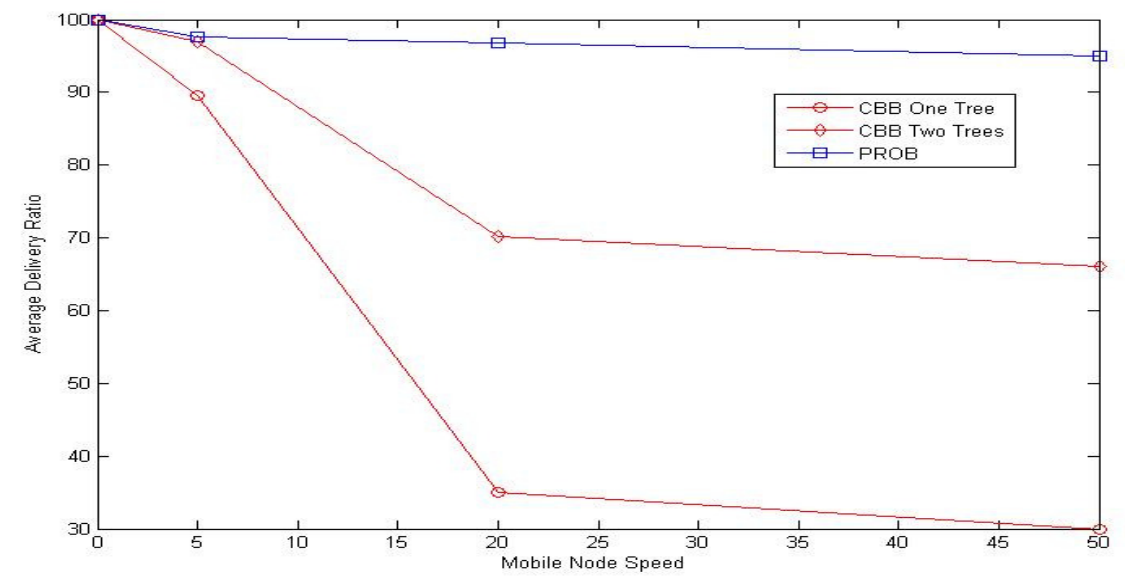

Figure 6: Delivery Ratio for Density=2 versus Mobility

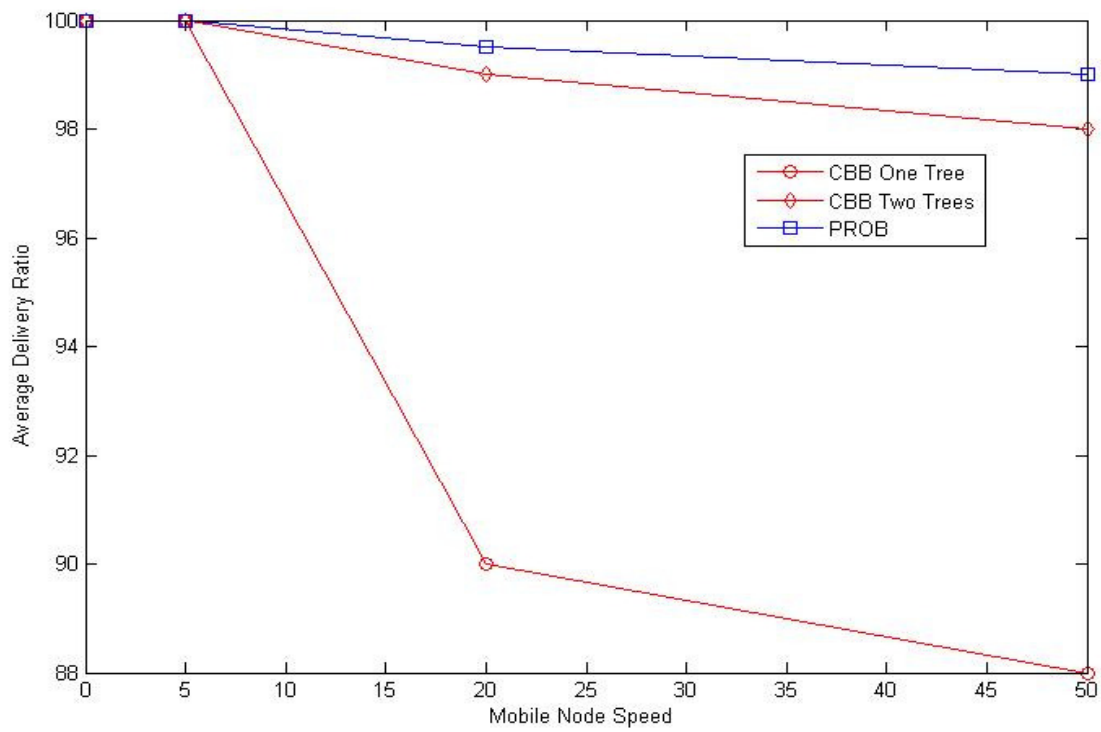

Figure 7: Delivery Ratio for Density=4 versus Mobility

From figures 5-7 we can see that first, for mobility zero all three protocols (CBB one tree, $\mathrm{CBB}$ two trees and PROB) perform $100 \%$ delivery ratio even for low density $=1$. Figure 5 (density $=1$ ), shows that for relatively low mobility (from 5 to $10 \mathrm{Km} / \mathrm{H}$ ) both $\mathrm{CBB}$ one and two trees perform better than PROB. When the mobility is higher, PROB performs better. 
International journal on applications of graph theory in wireless ad hoc networks and sensor networks

(GRAPH-HOC) Vol.3, No.4, December 2011

From figure 6 (density=2) we can see that for mobility from 0 to $5 \mathrm{Km} / \mathrm{H}, \mathrm{CBB}$ two tree and PROB have similar delivery ratio, but for higher mobility PROB performs better. Figure 7 $($ density=4) shows that both CBB two trees and PROB have close delivery ratio (greater than 99\%) for the different simulated mobility values. We also conducted simulation for density greater or equal to 5 , in these cases all the three protocols perform $100 \%$ delivery ratio.

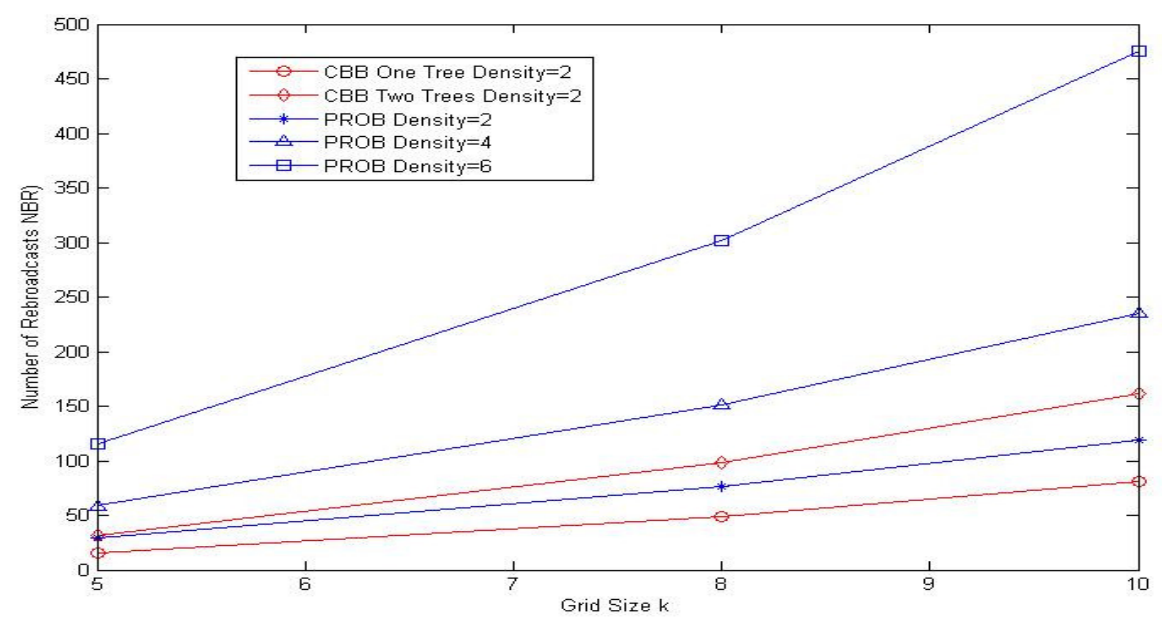

Figure 8: Simulation Results for NBR varying the Density and the Size

In other hand, figure 8, shows the superiority of $\mathrm{CBB}$ protocol, it has less rebroadcasting messages (less overhead per broadcast operation) compared to the probabilistic protocol PROB especially when the density and the size of the MANET increases. Note the close correspondence between the results our analytic results in figure 4 and the simulation results in figure 8 .

\section{CONClusion}

We have proposed a new approach for broadcasting in mobile ad-hoc networks called CBB protocols. $\mathrm{CBB}$ views the geographical region as a logical 2-dimensional grid. It uses a spanning tree of the grid cell, where each node of the spanning tree corresponds to the gateway node of that cell. Each node maintains a list of gateway nodes located in neighbouring grid cells for efficient packet rebroadcast. The maintenance of gateway nodes is based on normal traffic (unicast or broadcast) with no gateway election costs. Simulation results show that for dense networks $(D>=4) \mathrm{CBB}$ and $\mathrm{PROB}$ protocols have similar delivery ratio (99\%) even under relatively high mobility conditions. Our analytical study shows that for dense networks the number of rebroadcast messages $N B R$ of $\mathrm{CBB}$ is much lower than the one for PROB. That is because $N B R_{P R O B}$ is quadratic in terms of the network density. The simulation results confirm our analytical model for estimating the number of rebroadcast message for CBB protocol. It is known that designing a MANET protocol that performs well in all scenarios of different mobility and density conditions is not possible. We recommend CBB protocol whenever the MANET density is greater or equal to 4 because it has a high delivery ratio with less rebroadcast messages (fewer collisions at the data link layer) 
International journal on applications of graph theory in wireless ad hoc networks and sensor networks

(GRAPH-HOC) Vol.3, No.4, December 2011

\section{REFERENCES}

[1] I. Stojmenovic, Position-Based Routing in Ad Hoc Networks, IEEE Communications, July 2002, pp. 128-134.

[2] S. Giordano, I. Stojmenovic, and L. Blazevic, Position based routing algorithms for ad hoc networks: A taxonomy. In X. Cheng, X. Huang, and D. Du, editors, Ad Hoc Wireless Networking. Kluwer, December 2003.

[3] M. Mauve, J. Widmer and H. Hartenstein, A Survey on Position-Based Routing in Mobile Ad-Hoc Networks, IEEE Network Magazine, 15(6), November 2001, 30-39.

[4] S. Kamali and J. Opatrny, POSANT: A Position Based Ant Colony Routing Algorithm for Mobile Ad Hoc Networks, Journal of Networks, v. 3, no. 4, pp. 31-41, 2008.

[5] J. Hightower and G. Borriello, Location Systems for Ubiquitous Computing, Computer, 34(8), August 2001, 57-66.

[6] Jean-Claude Bermond, Philippe Michallon, Denis Trystram: Broadcasting in wraparound meshes with parallel monodirectional links. Parallel Computing 18(6): 639-648, 1992

[7] A. Touzene and Brigitte Plateau. Optimal multinode broadcast on a mesh connected graph with reduced bufferization. In Proceedings of the Second European Distributed Memory Computing Conference, 1991.

[8] B.Williams, T.Camp, "Comparison of Broadcasting Techniques for Mobile Ad Hoc Networks" Proceedings of the ACM Symposium on Mobile Ad Hoc Networking and Computing (MOBIHOC), 194-205, 2002.

[9] C. Ho, K. Obraczka, G. Tsudik, and K. Viswanath. Flooding for reliable multicast in multi-hop ad hoc networks. In Proceedings of the International Workshop on Discrete Algorithms and Methods for Mobile Computing and Communication (DIALM), pp. 64-71, 1999.

[10] J. Jetcheva, Y. Hu, D. Maltz, and D. Johnson. A simple protocol for multicast and broadcast in mobile ad hoc networks. Internet Draft: draft-ietf-manetsimple- mbcast-01.txt, July 2001.

[11] S. Ni, Y. Tseng, Y. Chen, and J. Sheu. The broadcast storm problem in a mobile ad hoc network. In Proceedings of the ACM/IEEE International Conference on Mobile Computing and Networking (MOBICOM), pp. 151-162, 1999.

[12] H. Lim and C. Kim. Multicast tree construction and flooding in wireless ad hoc networks. In Proceedings of the ACM International Workshop on Modeling, Analysis and Simulation of Wireless and Mobile Systems (MSWIM), 2000.

[13] W.-H. Liao, Y.-C. Tseng and J.-P. Sheu. Grid: A Fully Location-Aware Routing Protocol for Mobile Ad Hoc Networks, Telecommunication Systems, 18(1), 2001, pp. 37-60.

[14] S. Chinara and S. K. Rath., "A Survey on One-Hop Clustering Algorithms in Mobile Ad Hoc networks." Journal of Network System Managment, 2009, Vol. 17, pp. 183-207.

[15] M. Joa-Ng and I.-T. Lu, A peer-to-peer zone-based two-level link state routing for mobile ad hocnetworks, IEEE Journal on Selected Areas in Communications 17(8) (1999) 1415-1425.

[16] H. Hartenstein, C. Bettstetter, and X. Prez-Costa, "Stochastic Properties of the Random Waypoint Mobility Model," ACM/Kluwer Wireless Networks, special issue on modeling and analysis of mobile networks, 2004.

[17] H-W Tsai and T-S Chen, Service Discovery in Mobile Ad Hoc Networks Based on Grid. IEEE Transaction on Vehicular Technology, Vol. 58, No. 3, 2009. 